Pacific Journal of Mathematics

* PRODUCTS AND REPRESENTATIONS OF NILPOTENT 


\title{
* PRODUCTS AND REPRESENTATIONS OF NILPOTENT GROUPS
}

\author{
D. ARNAL
}

\begin{abstract}
On each orbit $W$ of the coadjoint representation of a nilpotent, connected and simply connected Lie group $G$, there exist * products which are relative quantizations for the Lie algebra $g$ of $G$. Choosing one of these * products, we first define a *-exponential for each $X$ in $\mathfrak{g}$. These *-exponentials are formal power series and, with the * product, they form a group. Thanks to that, we are able to define a representation of $G$ in a "* polarization" and to intertwine it with the unitary irreducible one associated to $W$. Finally, we study the uniqueness of our construction.
\end{abstract}

1. Introduction. The mathematical signification of quantization was specified by Bayen, Flato, Fronsdal, Lichnerowicz and Sternheimer with the theory of deformations of the associative algebra of $C^{\infty}$ functions on the symplectic manifold $W$ defined by the classical system [3]. (The principal results of the theory will be given in $§ 2$ for completeness.)

Previously, some other methods of geometrical quantization were considered by Kiriilov $[7,9,16,8]$. This last approach had a very important link with questions of finding and classifying unitary irreducible representations of a group $G$. The easiest and the most complete case is of course when $G$ is nilpotent. Let us suppose $G$ is nilpotent, connected and simply connected. We know all its unitary irreducible representations $[8$, 15]. There exists a one-to-one mapping between classes of these representations $U$ and orbits $W$ of the coadjoint representation of $G$. On the other hand, the geometrical quantization of $W$, i.e. the construction of fibre bundles with base $W$ and fibre a circle, is unique, the de Rham cohomology of $W$ being trivial. Moreover, the representation $U$ can be canonically defined with that quantization and a polarization [7].

It is tempting to "test" the method of quantization by deformation ( * quantization) on the problem of constructing and classifying unitary irreducible representations of connected, simply connected, nilpotent groups.

The goal of this work is to canonically find the unitary irreducible representation associated to an arbitrary orbit $W$ by means of $*$ products defined on $W$. We first recall the principal definitions and results of the theory of $*$ products (which are formal deformations with parameter $\lambda$ of 
the algebra $\left.C^{\infty}(W)\right)$. We consider the invariance and covariance of * products with respect to the action of $G$. Then we give an example showing that a too strong invariance property cannot be imposed. We thus consider only g-relative quantizations, i.e. * products for which the relation

$$
\tilde{X} * \tilde{Y}-\tilde{Y} * \tilde{X}=2 \lambda \widetilde{[X, Y]} \quad \forall X, Y \in \mathfrak{g}
$$

holds (here $\tilde{X}$ is the function defined on $W$ by $\tilde{X}(\xi)=\langle\xi, X\rangle, \xi \in W$ ).

We prove the existence of relative quantizations on each orbit $W$ : the so-called Moyal $*$ product defined in a particular global chart on $W$.

Now we study the representations property of that $*$ product. We remain in the frame of deformation theory as far as possible: we fix the value of $\lambda$ only in the last step. Thus our approach is entirely distinct from the method of Fronsdal and Lugo [4, 11]. Moreover, we do not limit the structure of $G$ and $W$.

For each $X$ in $g$, we can define a formal power series in $1 / \lambda$ :

$$
e^{* \tilde{X} / 2 \lambda}=\sum_{n>0} \frac{1}{n !}\left(\frac{1}{2 \lambda}\right)^{n}(\tilde{X})^{* n} .
$$

The set $G^{*}$ of such series is a group for the law *. The map

$$
\Phi: G \rightarrow G^{*}, \quad \Phi(\exp X)=e^{* \tilde{X} / 2 \lambda},
$$

is a group homomorphism.

Let $\mathfrak{n}$ be a subalgebra of $g$ subordinate to $\xi_{0}$ in $W$. Following a method of Fronsdal, we solve, in a space of formal power series in $1 / \lambda$, the equation

$$
x * \tilde{X}=\xi_{0}(X) x \quad \forall X \in \mathfrak{n} .
$$

The space $S$ of solutions ( * polarization) carries a representation $\pi$ of $G$ :

$$
\pi(g) x=\Phi(g) * x .
$$

We define an intertwining operator between $\pi$ and the unitary irreducible representation $U$ associated to $W$. This operator fixes the value of our parameter $\lambda$ and sums the formal series. That gives us each class of unitary irreducible representations for arbitrary nilpotent $G$.

In the last part we prove that $G^{*}$ and $\Phi$ are unique up to an automorphism and we study the uniqueness of $\pi$.

2. * Products. * products are introduced in [3] in order to define the quantization(s) of a problem of classical mechanics. In fact we suppose that quantum mechanics can be described as a deformation (in 
the sense of Gerstenhaber [5]) of classical mechanics with parameter $\lambda$ related to use $\hbar$ (in general $\lambda=i \hbar / 2$ ). We thus deform the structure of the set of observables without modifying the observables themselves. More precisely:

The classical problem is described by a symplectic manifold $W$; on the space $N=C^{\infty}(W, \mathbf{R})$ of observables, we consider the structures of Lie algebras (for the Poisson bracket $\{$,$\} ) and associative algebras (for usual$ product). $\mathrm{A} *$ product is an associative deformed structure

$$
u * v=u v+\sum_{r>0} \lambda^{r} C^{r}(u, v) \quad \forall u, v \in N
$$

( $u * v$ is a formal power series). We suppose that each $C^{r}$ is a bidifferential operator vanishing on constants. Thus the relation

$$
1 * u=u * 1=u, \quad \forall u \in N,
$$

holds. Of course we do not impose commutativity for $*$ since the * products of observables will correspond to composition of operators in the usual formalism of quantum mechanics. Gerstenhaber defined a cohomology associated to that deformation problem:

If $C$ is an $n$-differential operator, vanishing on constants, its coboundary $\delta C$ will be: $\forall u_{0}, \ldots, u_{n} \in N$

$(\delta C)\left(u_{0}, u_{1}, \ldots, u_{n}\right)$

$$
\begin{aligned}
= & u_{0} C\left(u_{1}, \ldots, u_{n}\right)-C\left(u_{0} u_{1}, u_{2}, \ldots, u_{n}\right)+C\left(u_{0}, u_{1} u_{2}, \ldots, u_{n}\right) \\
& +\cdots+(-1)^{n} C\left(u_{0}, u_{1}, \ldots, u_{n-1} u_{n}\right)+(-1)^{n+1} C\left(u_{0}, \ldots, u_{n-1}\right) u_{n} .
\end{aligned}
$$

Now if $\omega$ is an $n$-form on $W$, we define an $n$-differential operator $C_{\omega}$ by

$$
C_{\omega}\left(u_{1}, \ldots, u_{n}\right)=\omega\left(X_{u_{1}}, \ldots, X_{u_{n}}\right), \quad\left(u_{1}, \ldots, u_{n}\right) \in N,
$$

where $X_{u}$ is the symplectic gradient of $u$, i.e.,

$$
X_{u} v=\{u, v\} \quad \forall v \in N .
$$

J. Vey [17] determined the cohomology groups $H^{n}(\delta)$ :

$$
H^{n}(\delta)=\left\{\left[C_{\omega}\right], \omega n \text {-form on } W\right\} \simeq \text { space of } n \text {-forms on } W .
$$

The obstruction to deformation is in $H^{3}(\delta)$, the equivalence of deformations is given (in the theory of Gerstenhaber) by $H^{2}(\delta)$. These groups are in general very large. Now we want to simultaneously deform the Lie algebra structure of $N$ with the * commutator because usually the commutator of operators corresponds (up to an $i \hbar$ factor) to the Poisson 
bracket for classical observables. Thus we impose

$$
\begin{aligned}
& C^{1}(u, v)=\{u, v\}, \\
& C^{n}(u, v)=(-1)^{n} C^{n}(v, u), \quad \forall u, v \in N .
\end{aligned}
$$

Then

$$
\frac{1}{2 \lambda}(u * v-v * u)=[u, v]=\{u, v\}+\sum_{r>0} \lambda^{2 r} C^{2 r+1}(u, v)
$$

is a deformation of the Poisson bracket. The first condition is in fact admissible and the obstruction to deformation appears now as an element of $H^{3}(W)$, the third cohomology group in the de Rham cohomology of the manifold $W([13])$. Similarly, equivalences are described by $H^{2}(W)$ $([13]) ; *$ and $*^{\prime}$ are equivalents if there exists a series

$$
H=\mathrm{Id}+\sum_{r>0} \lambda^{r} H_{r}
$$

where the $H_{r}$ are differential operators without constant terms such that

$$
H(u * v)=H u *^{\prime} H v .
$$

3. Vey $*$ products. We know a $*$ product on $\mathbf{R}^{2 k}$, the so called Moyal $*$ product ([3]) associated to the Weyl-Wigner quantization

$$
u * v=u v+\sum_{n>0} \frac{\lambda^{n}}{n !} P^{n}(u, v)
$$

where the operators $P^{n}$ are defined by:

$$
\begin{aligned}
& P^{1}(u, v)=P(u, v)=\wedge^{l j} \partial_{i} u \partial_{j} v=\{u, v\}, \\
& P^{n}(u, v)=\bigwedge^{i_{1} j_{1}} \cdots \wedge^{l_{j} J_{n}} \partial_{l_{1} \cdots i_{n}} u \partial_{j_{1} \cdots j_{n}} v .
\end{aligned}
$$

If $W$ is a symplectic manifold and $\Gamma$ a symplectic connection on $W$, we can define operators

$$
P_{\Gamma}^{n}(u, v)=\bigwedge^{i_{1} j_{1}} \cdots \bigwedge^{i_{n} j_{n}} \nabla_{i_{1} \cdots i_{n}} u \nabla_{j_{1} \cdots j_{n}} v .
$$

But the series $\sum \lambda^{n} P_{\Gamma}^{n} / n$ ! defines an associative product only if $\Gamma$ is flat ([3]). By extension, we shall call a $*$ product a Vey * product if

$$
C^{n}(u, v)=\frac{1}{n !} Q^{n}(u, v) \text { for all } n,
$$

where $P_{\Gamma}^{n}$ and $Q^{n}$ have the same principal symbols (they do not depend on $\Gamma)$. Lichnerowicz [13] has shown:

THEOREM 3.1. Each * product is equivalent to a Vey * product. 
4. Invariant $*$ product. Let us now introduce a symplectic action of a Lie group $G$ on $W$. We have a notion of invariance.

Definition 4.1. $G$ acts on $N$ by

$$
(g \cdot u)(\xi)=u\left(g^{-1} \xi\right) \quad \forall u \in N, g \in G, \xi \in W .
$$

We extend, with the same notation, this action to the space of formal power series with coefficients in $N$.

$\mathrm{A} *$ product is invariant if

$$
g(u * v)=(g \cdot u) *(g \cdot v) \quad \forall u, v \in N, g \in G .
$$

Two $*$ products, $*$ and $*^{\prime}$, are equivariantly equivalents if they are equivalents:

$$
\left(\operatorname{Id}+\sum_{r>0} \lambda^{r} H_{r}\right)(u * v)=\left(\operatorname{Id}+\sum_{r>0} \lambda^{r} H_{r}\right) u *^{\prime}\left(\mathrm{Id}+\sum_{r>0} \lambda^{r} H_{r}\right) v
$$

and the $H_{r}$ are invariants.

In fact, the theory of invariant * products is well known only if there exists on $W$ an invariant connection. In the hermitian case S. Gutt [6] has shown the following theorem, whose generalization was given by Molin [12].

THEOREM 4.1. If there exists on $W$ an invariant connection, then the obstruction to constructing an invariant * product is in $H_{\mathrm{inv}}^{3}(W)$, the group of closed invariant 3-forms modulo exact invariant 3-forms, and the classification up to an invariant equivalence is described by $H_{\mathrm{inv}}^{2}(W)$.

On the other hand, the proof of Theorem 3.1 can be rewritten in the invariant case. We obtain

Proposition 4.1. If there exists on $W$ an invariant connection, then each invariant * product is equivariantly equivalent to an invariant Vey * product.

Finally, studying $C^{2}(u, v)$ it is easy to prove

Proposition 4.2. ([10]). If there exists on $W$ an invariant Vey * product, then there exists an invariant connection. 
5. A counterexample in the nilpotent case. From now on $G$ is a nilpotent, connected and simply connected Lie group, $g$ its Lie algebra, and $W$ one of their orbits in the coadjoint representation in $\mathrm{g}^{*}$, the dual of g. $W$ is a symplectic manifold on which $G$ acts. It is natural to ask for invariant $*$ products on $W$. Unfortunately this is generally impossible, as the following example shows:

Proposition 5.1. Let $\mathfrak{g}$ be the nilpotent Lie algebra with basis $X_{0}, X_{1}, \ldots, X_{n}, Y(n \geq 3)$ and commutation relations

$$
\left[Y, X_{l}\right]=X_{i-1}, \quad i=1, \ldots, n,
$$

all the remaining brackets vanishing. Let $G$ be the corresponding connected and simply connected Lie group. Then the generic orbits $W$ (the orbits such that $\left.\left\langle\xi, X_{0}\right\rangle \neq 0\right)$ are two dimensional, and there does not exist an invariant connection, neither an invariant * product, on $W$.

Proof. We easily show that $W$ can be parametrized by $(p, q) \in \mathbf{R}^{2}$ in such a manner that the vector fields differentials of the action of $G$ are

$$
q^{l} \frac{\partial}{\partial p}, \quad i=0,1, \ldots, n-1 ; \quad \frac{\partial}{\partial q}
$$

(see [2] and a general proof in Proposition 6.1). If $\Gamma$ is an invariant connection,

$$
\left[\partial_{p}, \nabla_{\partial_{q}} \partial_{q}\right]=0=\left[\partial_{q}, \nabla_{\partial_{q}} \partial_{q}\right]
$$

gives us

$$
\nabla_{\partial_{q}} \partial_{q}=a \partial_{p}+b \partial_{q} \quad \text { with } a \text { and } b \text { constants. }
$$

Moreover the following relations are incompatible:

$$
\begin{aligned}
{\left[q \partial_{p}, \nabla_{\partial_{q}} \partial_{q}\right] } & =-\nabla_{\partial_{p}} \partial_{q}-\nabla_{\partial_{q}} \partial_{p}=-b \partial_{p}, \\
{\left[q^{2} \partial_{p}, \nabla_{\partial_{q}} \partial_{q}\right] } & =-2 q\left(\nabla_{\partial_{p}} \partial_{q}+\nabla_{\partial_{q}} \partial_{p}\right)-2 \partial_{p}=-2 b q \partial_{p} .
\end{aligned}
$$

Let $G_{0}$ be the subgroup of $G$, exponential of $\partial / \partial q, \partial / \partial p$ and $q(\partial / \partial p)$. Clearly there exist $G_{0}$-invariant connections on $W$. Moreover a differential operator $H$ is $G_{0}$-invariant if and only if it is $G$-invariant.

Then if $*$ is a $G$-invariant $*$ product, it is a $G_{0}$-invariant $*$ product; there exists a $G_{0}$-invariant Vey $*$ product equivariantly equivalent to $*$. The equivalence being $G$-invariant, our Vey * product is $G$-invariant and there should be a $G$-invariant connection on $W$. 
6. Relative quantizations. The notion of relative quantization was given in [3]. Let $W$ be a coadjoint orbit of a Lie group $G$. Thus elements $X$ of $g$ appear as functions $\tilde{X}$ on $W$ :

$$
\tilde{X}(\xi)=\langle\xi, X\rangle \quad \forall \xi \in W, \forall X \in \mathfrak{g} .
$$

A g-relative quantization on $W$ is a * product such that

$$
[\tilde{X}, \tilde{Y}]=\frac{1}{2 \lambda}(\tilde{X} * \tilde{Y}-\tilde{Y} * \tilde{X})=\{\tilde{X}, \tilde{Y}\}(=\widetilde{[X, Y]}) \quad \forall X, Y \in \mathfrak{g} .
$$

In [2] we proved that each relative quantization is a covariant $*$ product. That means there exists a representation $\rho$ of $G$, by automorphisms of $*$, which is a deformation of the action defined in Definition 4.1:

$$
\begin{aligned}
& \rho(g)(u * v)=\rho(g) u * \rho(g) v, \\
& \rho\left(g g^{\prime}\right)=\rho(g) \rho\left(g^{\prime}\right), \\
& \rho(g)=\left(\operatorname{Id}+\sum_{s \geq 1} a_{s}(g)\right) \circ g,
\end{aligned}
$$

where the $a_{s}(g)$ are differential operators without constant terms. Thus relative quantizations give rise to representations. In the nilpotent case, we proved in [2] the existence of relative quantizations on each coadjoint orbit. For completeness and because it will be our starting point, we give this construction.

Proposition 6.1. ([15], [2]). Let $W$ be an orbit of the coadjoint representation of a nilpotent, connected and simply connected Lie group $G$. Then there exists on $W$ a global chart

$$
W \rightarrow \mathbf{R}^{2 k}, \quad \xi \mapsto(p, q),
$$

such that:

(a) The canonical 2-form on $W$ is $\sum_{t=1}^{k} d p_{\imath} \wedge d q_{i}$;

(b) Each $\tilde{X}, X \in \mathfrak{g}$ takes the form

$$
\tilde{X}(p, q)=\sum_{i=1}^{k} \alpha_{i}(q) p_{i}+\alpha_{0}(q)
$$

where the $\alpha_{i}(q)(i \geq 0)$ are polynomial functions in $q_{i+1}, \ldots, q_{k}$.

Proof. We prove it by induction on $\operatorname{dim} g$. Let $z$ be the center of $g$. If the kernel of the form $\mathfrak{m} \in z^{*}$ defined by

$$
\mathfrak{w}(Z)=\langle\xi, Z\rangle \quad \forall \xi \in W, Z \in \mathfrak{z},
$$


is nontrivial, $W$ is isomorphic to an orbit $\bar{W}$ of $\overline{\mathfrak{g}}=\mathfrak{g} / \mathrm{Ker} \mathfrak{w}$ and the proposition holds. If it is not the case, $z$ is one dimensional, and we can write

$$
\mathfrak{g}=\mathbf{R} X_{0}+\mathfrak{g}_{1},
$$

where $\mathfrak{g}_{1}$ is an ideal ([15]) and $W$ is isomorphic to $W_{1} \times \mathbf{R}^{2}$, where $W_{1}$ is an orbit in $\mathrm{g}_{1}^{*} \simeq X_{0}^{\perp}$, the isomorphism being

$$
\begin{gathered}
\left(\xi_{1}, q_{k}, p_{k}\right) \mapsto \exp -q_{k} X_{0} \xi_{1}+p_{k} \xi_{X_{0}}, \\
\xi_{X_{0}} \in g_{1}^{\perp} \text { and }\left\langle\xi_{X_{0}}, X_{0}\right\rangle=1 .
\end{gathered}
$$

This isomorphism gives us the chart of Proposition 6.1. A direct computation proves (a) and (b). The following corollary is an immediate consequence.

COROLlaRY 6.1. On each orbit $W$ of the coadjoint representation of $G$, the Moyal * product in the chart of Proposition 6.1 defines a relative quantization.

7. The $*$ exponential. The relation of relative quantization is formally

$$
\left(\frac{1}{2 \lambda} \tilde{X}\right) *\left(\frac{1}{2 \lambda} \tilde{Y}\right)-\left(\frac{1}{2 \lambda} \tilde{Y}\right) *\left(\frac{1}{2 \lambda} \tilde{X}\right)=\frac{1}{2 \lambda}[\overline{X, Y}] .
$$

Thus it is tempting to consider the functions $\tilde{X} / 2 \lambda$ in order to define a representation of $g$ and of $G$ by exponentiation. Let us be more precise. We now fix the chart of Proposition 6.1, put $p_{0}=1$ by convention and define the spaces:

$$
\begin{gathered}
A=\left\{\begin{array}{c}
\text { polynomial functions } x=\sum_{l=0}^{L}\left(\frac{1}{2 \lambda}\right)^{l} x_{l}, \\
\text { where } x=\sum_{i_{1} \cdots i_{l}} \alpha_{i_{1} \cdots i_{l}}(q) p_{l_{1}} \cdots p_{i_{l}}, \\
\alpha_{i_{1} \cdots i_{l}} \text { being a polynomial function } \\
\text { in the variables } \left.q_{j}, j>\inf \left(i_{1} \cdots i_{l}\right)\right\} ; \\
B=\left\{\text { formal power series } x=\sum_{l \geq 0}\left(\frac{1}{2 \lambda}\right)^{l} x_{l}\right. \\
\text { with the same conditions on } \left.x_{l}\right\} .
\end{array}\right.
\end{gathered}
$$

Now we take the Moyal * product on our chart. First we prove the existence of $\mathrm{a} *$ exponential. 
THEOREM 7.1. (a) For each $X_{1}, \ldots, X_{n}$ in $\mathfrak{g},(1 / 2 \lambda)^{n} \tilde{X}_{1} * \cdots * \tilde{X}_{n}$ belongs to $A$.

(b) The coefficient of $(1 / 2 \lambda)^{l}$ of this expression vanishes if $n$ is sufficiently large.

(c) For each $X$ in $\mathfrak{g}$, there exists a formal power series in $B$ :

$$
e^{* \tilde{X} / 2 \lambda}=\sum_{n \geq 0} \frac{1}{n !}\left(\frac{1}{2 \lambda}\right)^{n} \tilde{X}^{* n}
$$

Proof. (a) Starting from the relation

$$
\begin{aligned}
\frac{1}{2 \lambda} \tilde{X} * x= & \sum_{s \geq-1} \sum_{l=0}^{L}(2 \lambda)^{-l+s} \frac{1}{2^{s+1}(s+1) !} P^{s+1} \\
& \times\left(\sum_{i=0}^{k} \alpha_{i}(q) p_{l}, \sum_{i_{1} \cdots i_{l}} \alpha_{i_{1} \cdots i_{l}}(q) p_{l_{1}} \cdots p_{i_{l}}\right)
\end{aligned}
$$

where $X$ belongs to $g$ and $x$ to $A$, we see that the only remaining terms satisfy $s<l$ and are of the form $b_{i_{1} \cdots i_{l}} p_{i_{1}} \cdots p_{l_{l}}, b_{l_{2} \cdots i_{l}}$, being a polynomial function in the variables $q_{j}, j>\inf \left(i_{1} \cdots i_{l^{\prime}}\right) . X / 2 \lambda * A$ is thus in $A$.

(b) Let $r$ be the supremum of the degrees of $\alpha$ for all $X, X \in \mathfrak{g}$. We compute the coefficient of $(1 / 2 \lambda)^{l}$ in our expression. We find a linear combination of terms of the form

$$
D_{1}\left(\alpha_{J_{1}}\right) \cdot D_{2}\left(\alpha_{j_{2} 2}\right) \cdots D_{n}\left(\alpha_{j_{n} n}\right) p_{\iota_{1}} \cdots p_{\iota_{l}},
$$

where $\tilde{X}_{i}=\sum_{j} \alpha_{j i}(q) p_{j}$ and the $D_{i}$ are differential operators with constant coefficients. Let $i$ be the inf of $j_{1} \cdots j_{n}$. There does not exist a derivation in the variable $q_{i}$ in $D_{1}, \ldots, D_{n}$; hence we do not derive by $p_{i}$ (see the definition of $P^{r}$ ). Thus:

$$
\text { number of } s \text { such that } i_{s}=i \leq l \text {. }
$$

Considering successively the variables $q_{l+1}, q_{i+2}, \ldots, q_{k}$ we easily find:

$$
\text { number of } s \text { such that } i_{s}=i+t \leq l(r+1)^{t} \text {, }
$$

where $r$ is the supremum of $d^{0} \tilde{X}$ for all $X$ in $g$. It means that

$$
n \leq l\left[1+(r+1)+\cdots+(r+1)^{k}\right],
$$

which proves (b). (c) is an easy consequence of (b). In fact, our * exponential is a group morphism. 
THEOREM 7.2. (a) Let $G^{*}$ be the subset of $B$ defined by

$$
G^{*}=\left\{x \in B: \exists X \text { in } \mathfrak{g} \text { such that } x=e^{* \tilde{X} / 2 \lambda}\right\} .
$$

Then we can define the product $x * y$ of two elements of $G^{*}$, and $G^{*}$ with that product is a group.

(b) The map $\Phi: G \rightarrow G^{*}$ defined by

$$
\Phi(\exp X)=e^{* \tilde{X} / 2 \lambda}
$$

is a morphism from $G$ onto $G^{*}$.

Proof. Let $X_{1}, X_{2}, \ldots, X_{n} \in \mathfrak{g}$. In $B$ the series

$$
\begin{array}{r}
\sum_{m_{1} \geq 0}\left(\frac{1}{2 \lambda}\right)^{m_{1}} \frac{1}{m_{1} !} \tilde{X}_{1}^{* m_{1}} * \sum_{m_{2} \geq 0}\left(\frac{1}{2 \lambda}\right)^{m_{2}} \frac{1}{m_{2} !} \tilde{X}_{2}^{* m_{2}} \\
* \cdots * \sum_{m_{n} \geq 0}\left(\frac{1}{2 \lambda}\right)^{m_{n}} \frac{1}{m_{n} !} \tilde{X}_{n}^{* n}
\end{array}
$$

converges (as a formal power series). Indeed the coefficient of $(1 / 2 \lambda)^{l}$ depends only on the beginning of each series $e^{* \tilde{X}_{t} / 2 \lambda}$. The subset $C$ of $B$ of all these expressions is thus stable for the $*$ product.

Let us now consider the map $\Phi$. We shall prove that $\Phi$ is a morphism from $G$ to $C$ by induction on $\operatorname{dim} g$. Let $X_{1} \cdots X_{l}$ be a Jordan Hölder basis of $\mathfrak{g}$. We define

$$
\Psi\left(\exp t_{1} X_{1} \cdots \exp t_{l} X_{l}\right)=e^{* t_{1} \tilde{X}_{1} / 2 \lambda} * \cdots * e^{* t_{l} \tilde{X}_{l} / 2 \lambda} .
$$

We suppose that $\Psi$ is a morphism and $\Psi=\Phi$ if $\operatorname{dim} g<l$. We thus consider the subgroup $G_{1}$ of $G$ with Lie algebra $g_{1}$ generated by $X_{1} \cdots$ $X_{l-1}$.

We verify directly the relation

$$
e^{* \tilde{X} / 2 \lambda} * \tilde{Y} * e^{*-\tilde{X} / 2 \lambda}=\overparen{\operatorname{Ad} \exp (X)(Y)} \quad \forall X, Y \in \mathfrak{g}
$$

with these hypotheses and compute

$$
\begin{aligned}
\Psi\left(\exp X \exp t_{l} X_{l} \exp X^{\prime} \exp t_{l}^{\prime} X_{l}\right) \\
=\Psi\left(\exp X \exp \left(\operatorname{Ad} \exp t_{l} X_{l}\left(X^{\prime}\right)\right) \exp \left(t_{l}+t_{l}^{\prime}\right) X_{l}\right) \\
=\Phi(\exp X) * \Phi\left(\exp \left(\operatorname{Ad} \exp t_{l} X_{l} X^{\prime}\right)\right) * e^{*\left(t_{l}+t_{l}^{\prime}\right) \tilde{X}_{l}} \\
=\Phi(\exp X) * e^{* t_{l} \tilde{X}_{l}} * \Phi\left(\exp X^{\prime}\right) * e^{* t_{l}^{\prime} X_{l}} \\
=\Psi\left(\exp X \exp t_{l} X_{l}\right) * \Psi\left(\exp X^{\prime} \exp t_{l}^{\prime} X_{l}\right), \quad X, X^{\prime} \in \mathfrak{g}_{1} .
\end{aligned}
$$


Finally if $X=\sum a_{l} X_{i}$ belongs to $g$, we can write

$$
\exp t X=\exp \alpha_{1}(t) X_{1} \cdots \exp \alpha_{l}(t) X_{l}
$$

where each $\alpha_{i}$ is a polynomial function and $\partial \alpha_{i} /\left.\partial t\right|_{t=0}=a_{l}, \alpha_{i}(0)=0$ ([15]). $\Psi(\exp t X)$ is thus a formal power series with polynomial coefficients in $t$. By differentiation,

$$
\left.\frac{\partial}{\partial t} \Psi(\exp X)\right|_{t=0}=\frac{1}{2 \lambda} \tilde{X}
$$

and since $\Psi$ is a morphism,

$$
\frac{\partial}{\partial t}\left(\Psi(\exp X) * e^{*-\tilde{X} / 2 \lambda}\right)=0 \text {. }
$$

That proves that $\Psi=\Phi$ and $C=G^{*} . G^{*}$ is thus a group for $*$.

REMARKs. (a) In $\S 10$ we prove that $G^{*}$ does not depend on the choice of the chart 6.1.

(b) It is easy to add variables $p_{0}, q_{0}$ to $W$, and then consider direct products of orbits $W_{i}$ in such a manner that each $\tilde{X}$ is nonconstant on $\Pi_{i} W_{l}=W$. Thus on $W$ our computation gives an isomorphism of groups between $G$ and $G^{*}$ and we are able to find the Campbell-Hausdorff formula.

\section{A $*$ polarization and its representation.}

DeFinition 8.1. A subalgebra $\mathfrak{n}$ of $\mathfrak{g}$ is subordinate to $\xi \in W$ if

$$
\langle\xi,[\mathfrak{n}, \mathfrak{n}]\rangle=0
$$

The unitary irreducible representation of $G$ associated to $W$ is constructed from such an algebra. We easily see that:

LEMMA 8.1. With our notation, the algebra

$$
\mathfrak{n}=\left\{X \in \mathfrak{g} \text { such that } \tilde{X}(p, 0)=\tilde{X}(0,0) \forall p=\left(p_{1} \cdots p_{k}\right)\right\}
$$

is subordinate to $(0,0)$ and maximal.

The reader can easily prove that lemma by induction on $\operatorname{dim} \mathrm{g}$. We recall that $k\left(=\frac{1}{2} \operatorname{dim} W\right)$ is then the codimensionality of $\mathfrak{n}$ in $\mathrm{g}$. Starting 
with this subalgebra, adjusted to our chart, we define a $*$ polarization, following an idea of Fronsdal ([4]):

DEFINITION 8.2. We call polarization with respect to $n$ the space $S$ of all $x$ in $B$ such that

$$
x * \frac{1}{2 \lambda} \tilde{X}=\frac{1}{2 \lambda} \tilde{X}(0,0) \cdot x \quad \forall X \in \mathfrak{n}
$$

In fact, we will define (see Theorem 8.1) a representation of $G$ on $S, G$ acting on the left as in induced representations. Let us determine $S$ :

Proposition 8.1. If $\mathrm{n}$ is the algebra defined in Lemma 8.1, then:

(a) $x \in S$ if and only if

$$
x * q_{i}=0 \quad \forall i=1,2, \ldots, k .
$$

(b) $x \in S$ if and only if

$$
\begin{aligned}
x & =\sum_{r \geq 0}\left(\frac{1}{\lambda}\right)^{r+1} \sum_{s=0}^{r} \frac{(-1)^{s}}{s !}\left(\sum_{l=1}^{k} p_{l} q_{i}\right)^{s} a_{r-s}(q)\left(\frac{p_{0}}{2}\right)^{r-s+1} \\
& =e^{-p q / \lambda} \sum_{t \geq 0} a_{t}(q)\left(\frac{p_{0}}{2 \lambda}\right)^{t+1}
\end{aligned}
$$

Proof. (a) $\tilde{X}=q_{k}$ is in $\mathfrak{n}$ by construction ([15]). Thus $x * q_{k}$ has to vanish if $x$ is in $S$. But there exists in $n$ an element

$$
\tilde{X}=q_{k-1}+\sum_{l>0}\left(q_{k}\right)^{l} \sum_{i=1}^{k-1} \alpha_{l l}(q) p_{l},
$$

where $\alpha_{l l} \in \mathbf{R}\left[q_{i+1}, \ldots, q_{k-1}\right]$. Then $x * q_{k-1}$ also vanish. Thus, we show inductively that for each $x$ in $S$ the relations

$$
x * q_{i}=0, \quad \forall i=1, \ldots, k,
$$

hold. The converse is clear.

(b) Of course, the element

$$
e^{-p q / \lambda} \sum_{t \geq 0} a_{t}(q)\left(\frac{p_{0}}{2 \lambda}\right)^{t+1}
$$


is in $S$. Conversely, the relations

$$
x * q_{j}=\left(\sum_{n \geq 0} \lambda^{-n} x_{n}\right) * q_{j}=0, \quad \forall j=1, \ldots, k,
$$

are equivalent to

$$
x_{n} q_{j}=-\frac{\partial x_{n+1}}{\partial p_{j}}, \quad n \geq 0, \quad \forall j=1, \ldots, k .
$$

But they impose

$$
x_{0}=0, \quad x_{1}=a_{0}(q) p_{0},
$$

and if we suppose the existence of $a_{0}, \ldots, a_{r-1}$ such that

$$
x_{r}=\sum_{s=0}^{r-1} \frac{(-1)^{s}}{s !}\left(\sum p_{i} q_{l}\right)^{s} a_{r-s-1}(q) p_{0}^{r-s}
$$

let us put

$$
y_{r+1}=\sum_{s=1}^{r} \frac{(-1)^{s}}{s !}\left(\sum p_{i} q_{i}\right)^{s} a_{r-s}(q) p_{0}^{r-s+1}
$$

We obtain

$$
\frac{\partial}{\partial p_{j}}\left(y_{r+1}-x_{r+1}\right)=0 \text { for all } j
$$

or

$$
x_{r+1}=y_{r+1}+a_{n}(q) p_{0}^{r+1} \text {. }
$$

THEOREM 8.1. If $x$ belongs to $S$ and $g$ to $G$, then $\Phi(g) * x$ is well defined and belongs to $S$. We can define on $S$ a representation $\pi$ of $G$ by

$$
\pi(g) x=\Phi(g) * x .
$$

Proof. Keeping all our notations, we compute $1 / 2 \lambda \tilde{X} * x$ for $x$ in $S$ and $\tilde{X}=\sum_{l \geq 0} \alpha_{i}(q) p_{i}$ :

$$
\frac{1}{2 \lambda} \tilde{X} * x=e^{-p q / \lambda} \sum_{t \geq 0}\left(\frac{p_{0}}{2 \lambda}\right)^{t+1}\left(\frac{1}{2} \sum_{i>0} \alpha_{l}(2 q) \frac{\partial a_{t}}{\partial q_{i}}+\frac{p_{0}}{2 \lambda} \alpha_{0}(2 q) a_{t}\right)
$$


From that, we deduce that $\tilde{X} / 2 \lambda * x$ is in $S$ and the coefficient of $(1 / 2 \lambda)^{l}$ in the series $\tilde{X}_{1} / 2 \lambda * \tilde{X}_{2} / 2 \lambda * \cdots * \tilde{X}_{n} / 2 \lambda * x$ vanishes if $n$ is sufficiently large. $\Phi(\exp X) * x$ converges in the topology of formal power series and belongs to $S$ by construction. $\pi$ is of course a representation.

Our next step is to intertwine $\pi$ with the unitary irreducible representation associated to $W$. For that, we have to determine $\pi$ more precisely.

LEMMA 8.2. Let $X$ be an element of $g$ such that

$$
\tilde{X}=\sum_{i \geq 0} \alpha_{i}(q) p_{l}
$$

Let us define on $\mathbf{R}^{k}$ the vector field

$$
X^{-}=\sum_{i>0} \alpha_{l}(2 q) \frac{1}{2} \frac{\partial}{\partial q_{l}} .
$$

Then $X^{-}$is a complete vector field and its flow exp $-t X^{-}$has the form

$$
\left(\exp -t X^{-} \cdot q\right)_{j}=q_{j}-B_{j}\left(t ; q_{j+1}, \ldots, q_{k}\right)
$$

where $B_{j}$ is polynomial.

Proof. Computing successively $\left(\exp -t X^{-} \cdot q\right){ }_{j}$ for $j=k, k-1, \ldots, 1$, we find

$$
\left(\exp -t X^{-} \cdot q\right)_{J}=q_{J}-B_{j}\left(t ; q_{J+1}, \ldots, q_{k}\right)
$$

where

$$
\begin{aligned}
& B_{j}\left(t ; q_{j+1}, \ldots, q_{k}\right) \\
& \quad=\int_{0}^{t} \frac{1}{2} \alpha_{j}\left(2\left(q_{j+1}-B_{j+1}(s ; q)\right), \ldots, 2\left(q_{k}-B_{k}(s)\right)\right) d s .
\end{aligned}
$$

Proposition 8.2. Let

$$
x=e^{-p q / \lambda} \sum_{t \geq 0} a_{t}(q)\left(\frac{p_{0}}{2 \lambda}\right)^{t+1}
$$

be an element of $S$ and $X$ an element of $\mathfrak{g}$ such that

$$
\tilde{X}=\sum_{i \geq 0} \alpha_{i}(q) p_{i}
$$


Then

$$
\begin{aligned}
\pi(\exp X) x= & e^{-p q / \lambda} \exp \left\{\frac{p_{0}}{2 \lambda} \int_{0}^{1} \alpha_{0}\left(2 \exp -s X^{-} \cdot q\right) d s\right\} \\
& \times \sum_{t \geq 0} a_{t}\left(\exp -X^{-} \cdot q\right)\left(\frac{p_{0}}{2 \lambda}\right)^{t+1}
\end{aligned}
$$

Let us remark that this last expression is well defined and in $S$, thanks to Lemma 8.2.

Proof. Put

$$
\begin{aligned}
\varphi(u, x)= & e^{-p q / \lambda} \exp \left\{\frac{p_{0}}{2 \lambda} \int_{0}^{1} u \alpha_{0}\left(2 \exp -s u X^{-} \cdot q\right) d s\right\} \\
& \times \sum_{t \geq 0} a_{t}\left(\exp -u X^{-} \cdot q\right)\left(\frac{p_{0}}{2 \lambda}\right)^{t+1}, \quad u \in \mathbf{R} .
\end{aligned}
$$

We define a formal power series element of $S$. We directly see

$$
\frac{\partial}{\partial u} \varphi(u, x)=\varphi\left(u, \frac{1}{2 \lambda} \tilde{X} * x\right)
$$

From that, we deduce

$$
\frac{\partial}{\partial u} \varphi(u, \pi(\exp -u X) \cdot x)=0 \quad \text { for all } u \text {. }
$$

Then

$$
\begin{aligned}
\varphi(1, x) & =\varphi(1, \pi(\exp -X) \pi(\exp X) \cdot x) \\
& =\varphi(0, \pi(\exp X) x)=\pi(\exp X) \cdot x
\end{aligned}
$$

9. The UIR $U$ and the intertwining operator. First we recall the description of the unitary irreducible representation $U$ associated to $W$.

Proposition 9.1. Let $U$ be the unitary irreducible representation associated to $W$. Then:

(a) $U$ is induced by the character $\chi$ on $N=\exp n$ :

$$
\chi(\exp X)=e^{\sqrt{-1}\langle\xi, x\rangle} \quad \text { where } \xi=(0,0) .
$$

(b) The space $H$ where $U$ is defined is $L^{2}\left(\mathbf{R}^{k}\right)$ with variables $q_{i}$, $i=1, \ldots, k$, and Lebesgue measure. 
(c) The space $H^{\infty}$ of $C^{\infty}$ vectors for $U$ is the Schwartz space $\mathscr{S}$ of $C^{\infty}$ functions on $\mathbf{R}^{k}$, rapidly decreasing.

(d) For a good choice of variables $q$, if $X \in \mathfrak{g}$ is such that $\tilde{X}=$ $\sum_{i \geq 0} \alpha_{i}(q) p_{i}$, we can write

$$
(U(\exp X) f)(q)=\exp \left\{\sqrt{-1} \int_{0}^{1} \alpha_{0}\left(2 \exp -s X^{-} \cdot q\right) d s\right\} f\left(\exp -X^{-} \cdot q\right)
$$

( $X^{-}$is defined in Lemma 8.2).

Proof. (a)-(c) are well known (see [7] and [15] for instance). As usual, we prove (d) by induction on dim g. The only nontrivial case is when Ker $\mathfrak{w}=0$ (see proof of Proposition 6.1), then $U$ is induced by the representation $U_{1}$ of $G_{1}$ associated to $W_{1}([15])$. We introduce the variables $\tilde{q}=q_{1}, \ldots, q_{k-1}$ in $\mathbf{R}^{k-1}$. A function $f$ in the space $H$ of $U$ is a function from $\mathbf{R}$ with variables $q_{k}$ to $L^{2}\left(\mathbf{R}^{k-1}\right)$ with variables $\tilde{q}$. We identify $H$ to $L^{2}\left(\mathbf{R}^{k}\right)$. With this identification and the notations of the proof of Proposition 6.1, we have

$$
\begin{array}{r}
{\left[U\left(\exp X_{1} \exp t X_{0}\right) f\right]\left(q_{k}\right)=U_{1}\left(\exp \operatorname{Ad} \exp -q_{k} X_{0}\left(X_{1}\right)\right) \cdot f\left(q_{k}-t\right)} \\
=(\text { multiplier }) f\left(\left[\exp -\operatorname{Ad} \exp -q_{k} X_{0}\left(X_{1}\right)\right]^{-} \cdot \tilde{q}, q_{k}-t\right) .
\end{array}
$$

But we remark that

$$
\begin{array}{ll}
{\left[\left(\exp -t X_{0}^{-} \circ \exp -X_{1}^{-}\right) q\right]_{j}} & \\
\quad=\left[\left[\exp -\operatorname{Ad} \exp -q_{k} X_{0}\left(X_{1}\right)\right]^{-} \tilde{q}\right]_{j} & \text { if } j<k \\
\quad=q_{k}-t & \text { if } j=k .
\end{array}
$$

These relations are proved for $j=k$, then for $j=k-1, k-2, \ldots, 1$.

Up to the multiplier, (d) is proved. But the multiplier comes from $U_{1}$. Its form is

$$
\exp \left[\sqrt{-1} \int_{0}^{1} \alpha_{\circ q_{k}}\left(2\left[\exp -s \operatorname{Ad} \exp -q_{k} X_{0}\left(X_{1}\right)\right]^{-} \tilde{q}\right] d s\right)
$$

where, by definition, $\alpha_{\circ} q_{k}$ is the function of $q$ :

$$
\alpha_{\circ q_{k}}\left(q_{1}, \ldots, q_{k-1}\right)=\left[\operatorname{Ad} \exp -q_{k} X_{0}\left(X_{1}\right)\right]\left(0, \ldots, 0, q_{1}, \ldots, q_{k-1}, 0\right) \text {, }
$$

i.e. $\alpha_{\circ q_{k}}\left(q_{1}, \ldots, q_{k-1}\right)=\alpha_{0}\left(q_{1}, \ldots, q_{k}\right)$.

Now we define our intertwining operator $T$. Intuitively $T$ "fixes $p_{0} / 2 \lambda=\sqrt{-1}$ and multiplies $x$ by $e^{p q / \lambda}$ ".

Let us introduce the spaces $D$ and $V$ of $S$ and $L^{2}\left(\mathbf{R}^{k}\right)$ which are, respectively, the domain of $T$ and its range. 
LEMMA 9.1. Let $V$ be the subspace of $L^{2}\left(\mathbf{R}^{k}\right)$ defined by $V=\left\{f \in L^{2}\left(\mathbf{R}^{k}\right): f\right.$ is the restriction to $\mathbf{R}^{k}$ of an entire function $F$ on $\left.\mathbf{C}^{k}\right\}$. Let $V_{0}$ be $V \cap \mathscr{S}$. Then

(a) $V$ and $V_{0}$ are dense in $L^{2}\left(\mathbf{R}^{k}\right)$.

(b) $V$ and $V_{0}$ are stable under the action of $U(\exp X), \forall X \in \mathfrak{g}$.

(c) $V_{0}$ is stable under the action of $d U(X), X \in \mathfrak{g}$, and dense in $\mathscr{S}$ for the topology of $\mathscr{S}$.

Proof. (a) is obvious. Now we extend the operators $U(\exp X)$ to complex functions $F$ by

$$
(\bar{U}(\exp X) F)(z)=\exp \left\{\sqrt{-1} \int_{0}^{1} \alpha_{0}\left(2 \exp -s X^{-} \cdot z\right) d s\right\} F\left(\exp -X^{-} \cdot z\right),
$$

where $\exp -X^{-} \cdot z$ is the polynomial function extending $q \hookrightarrow \exp -X^{-} \cdot q$. Then $\bar{U}(\exp X) F$ is entire if and only if $F$ is entire and

$$
\left.\bar{U}(\exp X) F\right|_{\mathbf{R}^{k}}=U(\exp X)\left(\left.F\right|_{\mathbf{R}^{k}}\right) .
$$

This proves (b) for $V$; for $V_{0}, \mathscr{S}$ is stable since it is $H^{\infty}$.

(c) By construction the generator $d U(X)$ of the one parameter group $U(\exp t x)$ is a differential operator with polynomial coefficients. Then $d U(X)$ leaves $V_{0}$ invariant, and a classical result of Poulsen ([14]) proves (c).

Lemma 9.2. Let $D$ (resp. $D_{0}$ ) be the subspace of $S$ defined by the relations $x \in D$ (resp. $D_{0}$ ) if and only if there exists an integer $n, x_{1}, \ldots, x_{n}$ in $S$ and $X_{1}, \ldots, X_{n}$ in $\mathrm{g}$, such that:

(1) $x=\sum_{i=1}^{n} x_{i}$.

(2)

$$
\pi\left(\exp X_{i}\right) x_{i}=e^{-p q / \lambda} \sum_{t \geq 0} a_{t l}(q)\left(\frac{p_{0}}{2 \lambda}\right)^{t+1},
$$

where the polynomial functions $a_{t i}$ are homogeneous with degree $t$.

(3) $\sum_{t \geq 0}(\sqrt{-1})^{t+1} a_{t i}(z)$ is the development on $\mathbf{C}^{k}$ of an entire function $F_{i} \forall i=1, \ldots, n$. $L^{2}\left(\mathbf{R}^{k}\right)$,

(4) $f_{i}=F_{\left.i\right|_{\mathbf{R}^{h}}}$ belongs to $V$ (resp. $\left.V_{0}\right) \forall i=1, \ldots, n$. Then the vector of

$$
\sum_{i} U\left(\exp X_{i}\right) f_{l}
$$

depends on $x$ only. In particular it does not depend on the chosen decomposition (1). 
Proof. Let us extend $U$ to the representation $\bar{U}$ on $\mathscr{S}^{\prime}$ by putting

$$
\langle\bar{U}(\exp X) T, f\rangle=\langle T, U(\exp -X) f\rangle \quad \forall f \in \mathscr{S}, T \in \mathscr{S}^{\prime} .
$$

For each function $\varphi$ on $\mathbf{R}^{k}, C^{\infty}$ with compact support, we have

$$
\left\langle\sum_{i} U\left(\exp -X_{i}\right) f_{l}, \varphi\right\rangle=\sum_{i}\left\langle f_{i}, U\left(\exp X_{i}\right) \varphi\right\rangle
$$

Now $U\left(\exp X_{i}\right) \varphi$ has compact support, the development of $f_{i}$ converges uniformly on it, and we have

$$
\begin{aligned}
\left\langle\sum_{i} U\left(\exp -X_{i}\right) f_{i}, \varphi\right\rangle & =\sum_{i} \sum_{t \geq 0}\left\langle(\sqrt{-1})^{t+1} a_{t i}, U\left(\exp X_{i}\right) \varphi\right\rangle \\
& =\sum_{t \geq 0}\left\langle\sum_{i}(\sqrt{-1})^{t+1} U^{\prime}\left(\exp -X_{i}\right) a_{t i}, \varphi\right\rangle
\end{aligned}
$$

But we know $U$ and $\pi$ (see Propositions 9.1 and 8.2). Thus

$$
(\sqrt{-1})^{t+1} \sum_{i} \bar{U}\left(\exp -X_{i}\right) a_{t i}(q)=(\sqrt{-1})^{t+1} b_{t}(q) \text { for all } t
$$

where $b_{t}(q)$ is given by the development of $x$ :

$$
x=e^{p q / \lambda} \sum_{t \geq 0}\left(\frac{p_{0}}{2 \lambda}\right)^{t+1} b_{t}(q) .
$$

Thus $\left\langle\sum_{i} U\left(\exp -X_{i}\right) f_{i}, \varphi\right\rangle$ depends only on $x$.

Let us preserve our notations. We are now able to prove

THEOREM 9.1.

(a) $D$ and $D_{0}$ are $\pi$ stable subspaces of $S$.

(b) The operator $T: D \rightarrow V$, defined by

$$
T x=\sum_{i} U\left(\exp -X_{i}\right) f_{i},
$$

is a linear intertwining of $\pi$ and $U$ :

$$
T \pi(g)=U(g) T .
$$

(c) The range of $T$ is $V$.

(d) The conclusions of (b) and (c) hold with $T_{0}=\left.T\right|_{T_{0}}, D_{0}$ and $V_{0}$, respectively. 
Proof. The restriction to $D_{0}$ is obvious.

(a) $D$ is of course $\pi$ stable since if $x$ is in $D$,

$$
\pi(\exp X) x=\sum_{i} \pi(\exp X) x_{l}
$$

and the $\pi\left(\exp X_{i} \exp -X\right)\left(\pi(\exp X) x_{i}\right)$ satisfy conditions (2)-(4) of Lemma 9.2.

(b) Lemma 9.2 defines $T$ and, with that definition,

$$
\begin{aligned}
T \pi(\exp X) x & =\sum_{i} U\left(\exp X \exp -X_{i}\right) f_{i} \\
& =U(\exp X) T x \quad \forall x \in D, \forall X \in \mathfrak{g} .
\end{aligned}
$$

$T$ is linear by construction.

(c) For each $f$ in $V, f$ is the restriction to $\mathbf{R}^{k}$ of $\sum_{t \geq 0} a_{t}(z)(\sqrt{-1})^{t+1}$ and thus

$$
f=T x
$$

where $x$ is the element of $D$ :

$$
x=e^{-p q / \lambda} \sum_{t \geq 0}\left(\frac{p_{0}}{2 \lambda}\right)^{t+1} a_{t}(q) .
$$

10. Unicity. Until now we only considered a given chart for $W$. In this section we study the dependence of our construction on the choice of the chart in Proposition 6.1. First, we prove that $G^{*}$ is, up to isomorphism, unique. More precisely:

Proposition 10.1. Let $\xi \hookrightarrow(p, q)$ and $\xi \hookrightarrow\left(p^{\prime}, q^{\prime}\right)$ be two charts of $W$ constructed as in Proposition 6.1, and let $G^{*}$ and $G^{* \prime}$ be the two groups defined from these charts (see Theorem 7.2). Then the correspondence

$$
\Psi: G^{*} \rightarrow G^{* \prime}, \quad \Psi\left(e^{* \tilde{X} / 2 \lambda}\right)=e^{*^{\prime} \tilde{X} / 2 \lambda},
$$

is a group isomorphism.

Proof. Let us denote by $\Phi, \pi, U$ and $T$ the same objects as in previous sections for the first chart. In particular, $\Phi$ is the group morphism $\Phi$ : $G \rightarrow G^{*}$ defined by $\Phi(\exp X)=e^{* \tilde{X} / 2 \lambda}$.

Let us determine the kernel of $\Phi$. If $\Phi(\exp X)=1$ we can write

$$
\Phi(\exp t X)=\sum_{l \geq 0}\left(\frac{1}{2 \lambda}\right)^{l} x_{l}(t),
$$


where $x_{l}(t)$ is a polynomial function, since $x_{l}$ depends only on the first terms of the expansion of $\Phi(\exp X)$. For each integer $m, x_{l}(m)$ vanishes, since

$$
\Phi(\exp m X)=\Phi(\exp X) * \Phi(\exp X) * \cdots * \Phi(\exp X) .
$$

Then $\Phi(\exp t X)$ is identically equal to 1 . Now for each $x$ in $D$,

$$
T \pi(\exp t X) x=T(\Phi(\exp t X) * x)=T x=U(\exp t X) T x .
$$

Thus $T x$ belongs to the domain of $d U(X)$ and $d U(X) T x$ vanishes. That means, since $V$ is dense in $L^{2}\left(\mathbf{R}^{k}\right)$ and $d U(X)$ is a closed operator, that

$$
d U(X) \equiv 0 \text {. }
$$

Conversely, if $d U(X)$ is the null operator, it vanishes identically on $\mathscr{S}$ and on $\mathscr{S}^{\prime}$ by duality. Concerning the function $1 \in \mathscr{S}^{\prime}$, if $\tilde{X}=\sum \alpha_{i}(q) p_{i}$, we have

$$
d U(X) \cdot 1=\alpha_{0}(2 q) \equiv 0 .
$$

In the same way, concerning the function $q_{i} \in \mathscr{S}^{\prime}, i=1, \ldots, k$, we find

$$
d U(X) q_{l}=\frac{1}{2} \alpha_{i}(2 q) \equiv 0 .
$$

$\tilde{X}$ is the null function on $W$, and $\Phi(\exp X)$ is the function 1 . Let us now consider the kernel of our representation $U$ :

$$
\operatorname{Ker} U=\{g \in G: U(g)=\operatorname{Id}\} \text {. }
$$

We saw that $\operatorname{Ker} \Phi \subset \operatorname{Ker} U$. But the converse is also true since if $g$ belongs to $\operatorname{Ker} U, \bar{U}(g)$ defined on $\mathscr{S}^{\prime}$ is the identity operator and

$$
\bar{U}(g) \cdot 1=1
$$

means that our multiplier is 1 .

$$
\bar{U}(g) \cdot q_{i}=q_{i}
$$

means that $g=\exp X$, where the vector field $X^{-}$vanishes identically. Thus

$$
g=\exp X \quad \text { where } \tilde{X}=0
$$

We are now able to define an isomorphism $\Phi^{*}$ from $G / \operatorname{Ker} U$ to $G^{*}$. But for our second chart, the same holds: there exists an isomorphism $\Phi^{* \prime}$ from $G / \operatorname{Ker} U$ to $G^{* \prime}$. The proof is now complete since, by construction,

$$
\Psi=\Phi^{* \prime} \circ \Phi^{*-1} .
$$


The following example shows that $\pi$ is not uniquely defined. Of course the choice of a polarization in order to define $S$ and $\pi$ implies the nonuniqueness.

LEMMA 10.1. Let $\mathfrak{g}$ be the three-dimensional Heisenberg algebra: $\mathfrak{g}$ is generated by $X, Y$ and $Z$ with commutation relations $[X, Y]=Z$. Let $W$ be the two dimensional orbit in $\mathrm{g}^{*}$ such that $\tilde{Z}=1$. Then we choose the first chart

$$
\tilde{X}=p, \quad \tilde{Y}=q,
$$

and the second chart

$$
\tilde{X}=q^{\prime}, \quad \tilde{Y}=-p^{\prime} .
$$

We keep our above notations with "primes" for the second chart. Then if $x_{0}=e^{-p q / \lambda} p_{0} / 2 \lambda \in S$ we have

$$
\pi(\exp t X) x_{0}=x_{0} \text { for all } t,
$$

and in $S^{\prime}$ there does not exist an element $x_{0}^{\prime}$ such that

$$
\pi^{\prime}(\exp t X) x_{0}^{\prime}=x_{0}^{\prime} \quad(t \neq 0) .
$$

Thus there does not exist an isomorphism between $S$ and $S^{\prime}$ intertwining $\pi$ and $\pi^{\prime}$.

Proof. We recall that

$$
S=\left\{x \in B: x=e^{-p q / \lambda} \sum_{t \geq 0}\left(\frac{p_{0}}{2 \lambda}\right)^{t+1} a_{t}(q)\right\}
$$

and

$$
S^{\prime}=\left\{x \in B: x^{\prime}=e^{-p^{\prime} q^{\prime} / \lambda} \sum\left(\frac{p_{0}}{2 \lambda}\right)^{t+1} a_{t}\left(q^{\prime}\right)\right\}
$$

Thus

$$
\begin{aligned}
& \pi(\exp \tau X) x=e^{-p q / \lambda} \sum_{t \geq 0}\left(\frac{p_{0}}{2 \lambda}\right)^{t+1} a_{t}(q-\tau), \\
& \pi^{\prime}(\exp \tau X) x=e^{-p^{\prime} q^{\prime} / \lambda} e^{\left(-p_{0} / 2 \lambda\right) 2 q^{\prime} \tau} \sum_{t \geq 0}\left(\frac{p_{0}}{2 \lambda}\right) a_{t}\left(q^{\prime}\right) .
\end{aligned}
$$

The stabilizer of $x_{0}$ contains $\exp \tau X$ for each $\tau$, but the equation

$$
\pi^{\prime}(\exp \tau X) x=x \quad(\tau \neq 0)
$$

implies

$$
\pi^{\prime}(\exp \tau X) x=x \quad \forall \tau \quad \text { and } \quad \frac{p_{0}}{2 \lambda} 2 q^{\prime} \cdot x=0
$$


In fact the intertwining operator between $U$ and $U^{\prime}$ is the Fourier transform and constants in $\mathscr{S}^{\prime}$ are transformed in Dirac measures.

Nevertheless, we can define an intertwining operator between the two $g$ modules $S$ and $S^{\prime}$. Indeed in $V_{0}$ there exist some very particular functions (see [1]). As usual, we denote by $\pi, U, S, V_{0}$ the representations and space defined with a chart $(p, q)$ and by $\pi^{\prime}, U^{\prime}, S^{\prime}, V_{0}^{\prime}$ those associated to another chart $\left(p^{\prime}, q^{\prime}\right)$. The representations $U, \pi, U^{\prime}, \pi^{\prime}$ give rise by differentiation to representations $d U, d \pi, \ldots$ of $g$ and of its enveloping algebra $\mathscr{U}(\mathfrak{g})$. We define

$$
f_{0}(q)=\exp \left\{-\sum q_{j}^{2}+e^{-\sum q_{j}^{2}}\right\} \in V_{0} .
$$

$f_{0}^{\prime}$ is defined similarly in $V_{0}^{\prime}$ with variables $q^{\prime} . x_{0}$ is the element of $S$ defined by the development of the entire function $f_{0}: f_{0}=T x_{0} . x_{0}^{\prime}$ is in $S^{\prime}$ with a similar definition.

PROPOSITION 10.2.

(a) The g modules $d U(\mathscr{U}(\mathfrak{g})) f_{0}$ and $\Delta_{0}=d \pi(\mathscr{U}(\mathfrak{g})) x_{0}$ are isomorphic to

$$
\mathscr{U}(\mathfrak{g}) / \operatorname{Ker} d U
$$

(b) The operator

$$
\theta: \Delta_{0} \rightarrow \Delta_{0}^{\prime}, \quad d \pi(u) x_{0} \hookrightarrow d \pi^{\prime}(u) x_{0}^{\prime},
$$

is an intertwining operator of $\mathrm{g}$ modules.

Proof. The proof is very easy. In fact in [1] it is proved that

$$
d U(u) f_{0}=0 \text { implies } d U(u) \equiv 0 .
$$

Thus with $T$, the same property holds for $x_{0}$ and $d \pi$, and of course for $x_{0}^{\prime}$ and $d \pi^{\prime}$. This proves that $\theta$ is a well-defined isomorphism between $\Delta_{0}$ and $\Delta_{0}^{\prime}$.

Proposition 10.2 is somewhat unsatisfactory because as a g-module, $S$ is too rich. We can find in it many other $g$-submodules equivalent to the corresponding submodule in $S^{\prime}$.

Finally, let us remark that the notion of $*$ product is a geometrical one and depends essentially on $W$ itself, not on the choice of the duality which allows us to consider $W$ as an orbit in $g^{*}$. Thus the notion of the group $G^{*}$ is the canonical one. Polarizations are introduced in this context only in order to find (more or less) irreducible representations of $G$. As usual, see [4] for instance, we are able from the orbit $W$ to construct all 
unitary irreducible representations in $L^{2}\left(\mathbf{R}^{k}\right)$ with the same kernel. More precisely, let us choose such a representation $U^{\prime}$ induced by a character $\chi^{\prime}$ of the subgroup $N^{\prime}$. Let $W^{\prime}$ be the associated orbit, $\tilde{X}^{\prime}$ the function $\tilde{X}$ computed on $W^{\prime}$. We have

$$
e^{\sqrt{-1} \tilde{X}^{\prime}(0,0)}=\chi^{\prime}(\exp X) \quad \forall X \in \mathfrak{n}^{\prime},
$$

the Lie algebra of $N^{\prime}$. Since $\operatorname{Ker} U^{\prime}=\operatorname{Ker} U$, we see, as in the proof of Proposition 10.1, that

$$
\{X \in \mathfrak{g}: \tilde{X} \equiv 0 \text { on } W\}=\left\{X \in \mathfrak{g}: X^{\prime} \equiv 0 \text { on } W^{\prime}\right\} .
$$

Thus, the groups $G^{*}$ and $G^{* \prime}$ are isomorphic, and we define on $W$ the polarization

$$
S^{\prime \prime}=\left\{x \in B: \tilde{X} * x=\tilde{X}^{\prime}(0,0) \cdot x \forall X \in \mathfrak{n}^{\prime}\right\} .
$$

We define a representation $\pi^{\prime \prime}$ on $W$ and an intertwining operator between $\pi^{\prime \prime}$ and $U^{\prime}$ as in $\S 9$.

Acknowledgment. A part of this work was done during a stay at the Université Libre de Bruxelles, Belgium. The author thanks this University and the Solvay Institute for financial support.

I exposed some of these results in the weakly "séminaire de physique mathématique" to my colleagues at the University of Dijon. I thank them for their constant interest.

I am indebted to M. Cahen and S. Gutt for many fruitful exchanges of views.

M. Flato is at the origin of $*$ product theory. I thank him for many stimulating discussions on these and related questions.

\section{REFERENCES}

[1] D. Arnal, C. R. Acad. Sc. Paris, Série A, 284 (1977), 101.

[2] D. Arnal and al., Covariance and geometrical invariance in *-quantization, to appear in J.M.P.

[3] F. Bayen et al., Ann. of Phys., (N.Y.) 111, 61 and 111 (1978).

[4] C. Fronsdal, Rep. on Math. Phys., 15 (1979), 111.

[5] M. Gerstenhaber, Ann. of Math., 79 (1964), 59.

[6] S. Gutt, Déformations formelles de l'algèbres des fonctions différentiables sur une variété symplectique, Thesis. Université Libre de Bruxelles, (1980).

[7] A. A. Kirillov, Elements of the Theory of Representations, Springer-Verlag (Berlin) 1976.

[8] Unitary representations of the nilpotent Lie groups, (in Russian) Usp. Mat. Nauk., 17 (1962), 57-110. 
[9] B. Kostant, Lecture Notes in Mechanics, 170, Springer-Verlag (Berlin) 1970.

[10] A. Lichnerowicz, Deformations d'algèbres associées à une variété symplectique (les * produits), to appear in Ann. Inst. Fourier.

[11] V. A. Lugo, L.M.P. $5 \mathrm{n}^{\circ} 6,509$ (1981).

[12] P. Molin, Invariance et covariance de déformations de structures sur une variété symplectique, Thesis. Université de Dijon, 1981.

[13] O. M. Neroslavsky and A. T. Vlassov, C. R. Acad. Sc. Paris, Série I, 292 (1983), 71.

[14] N. S. Poulsen, J. of Funct. Anal., 9 (1972), 87.

[15] L. Pukanski, Leçons sur les représentations de groupes, Dunod (Paris), 1967.

[16] J. M. Souriau, Structure des systèmes dynamiques, Dunod (Paris), 1970.

[17] J. Vey, Comment. Math. Helv., 50 (1975), 421.

Received July 8, 1982 and in revised form January 1, 1983.

UNIVERSITE DE DiJON

BP 138

21004-DiJON, FRANCE 


\section{PACIFIC JOURNAL OF MATHEMATICS EDITORS}

\author{
DONALD BABBITT (Managing Editor) \\ University of California \\ Los Angeles, CA 90024 \\ J. DugundJI \\ University of Southern California \\ Los Angeles, CA 90089-1113 \\ R. FINN \\ Stanford University \\ Stanford, CA 94305 \\ HERMANN FLASChKa \\ University of Arizona \\ Tucson, AZ 85721
}

C. C. MOORE

University of California

Berkeley, CA 94720

Arthur Ogus

University of California

Berkeley, CA 94720

Hugo RossI

University of Utah

Salt Lake City, UT 84112

H. SAMELSON

Stanford University

Stanford, CA 94305

ASSOCIATE EDITORS
R. ARENS
E. F. BECKENBACH
B. H. NeUmanN
F. WOLF
K. YosHIDA (1906-1982)

\section{SUPPORTING INSTITUTIONS}
UNIVERSITY OF ARIZONA
UNIVERSITY OF BRITISH COLUMBIA
CALIFORNIA INSTITUTE OF TECHNOLOGY
UNIVERSITY OF CALIFORNIA
MONTANA STATE UNIVERSITY
UNIVERSITY OF NEVADA, RENO
NEW MEXICO STATE UNIVERSITY
OREGON STATE UNIVERSITY

\author{
UNIVERSITY OF OREGON \\ UNIVERSITY OF SOUTHERN CALIFORNIA \\ STANFORD UNIVERSITY \\ UNIVERSITY OF HAWAII \\ UNIVERSITY OF TOKYO \\ UNIVERSITY OF UTAH \\ WASHINGTON STATE UNIVERSITY \\ UNIVERSITY OF WASHINGTON
}

The Supporting Institutions listed above contribute to the cost of publication of this Journal, but they are not owners or publishers and have no responsibility for its content or policies.

Mathematical papers intended for publication in the Pacific Journal of Mathematics should be in typed form or offset-reproduced (not dittoed), double spaced with large margins. Please do not use built up fractions in the text of the manuscript. However, you may use them in the displayed equations. Underline Greek letters in red, German in green, and script in blue. The first paragraph must be capable of being used separately as a synopsis of the entire paper. In particular it should contain no bibliographic references. Please propose a heading for the odd numbered pages of less than 35 characters. Manuscripts, in triplicate, may be sent to any one of the editors. Please classify according to the scheme of Math. Reviews, Index to Vol. 39. Supply name and address of author to whom proofs should be sent. All other communications should be addressed to the managing editor, or Elaine Barth, University of California, Los Angeles, California 90024.

There are page-charges associated with articles appearing in the Pacific Journal of Mathematics. These charges are expected to be paid by the author's University, Government Agency or Company. If the author or authors do not have access to such Institutional support these charges are waived. Single authors will receive 50 free reprints; joint authors will receive a total of 100 free reprints. Additional copies may be obtained at cost in multiples of 50 .

The Pacific Journal of Mathematics is issued monthly as of January 1966. Regular subscription rate: $\$ 190.00$ a year (5 Vols., 10 issues). Special rate: $\$ 66.00$ a year to individual members of supporting institutions.

Subscriptions, orders for numbers issued in the last three calendar years, and changes of address should be sent to Pacific Journal of Mathematics, P.O. Box 969, Carmel Valley, CA 93924, U.S.A. Old back numbers obtainable from Kraus Periodicals Co., Route 100, Millwood, NY 10546.

The Pacific Journal of Mathematics at P.O. Box 969, Carmel Valley, CA 93924 (ISSN 0030-8730) publishes 5 volumes per year. Application to mail at Second-class postage rates is pending at Carmel Valley, California, and additional mailing offices. Postmaster: Send address changes to Pacific Journal of Mathematics, P.O. Box 969, Carmel Valley, CA 93924.

PUBLISHED BY PACIFIC JOURNAL OF MATHEMATICS, A NON-PROFIT CORPORATION

Copyright $\odot 1984$ by Pacific Journal of Mathematics 


\section{Pacific Journal of Mathematics}

Vol. 114, No. $2 \quad$ June, 1984

William Allen Adkins, A Harnack estimate for real normal surface

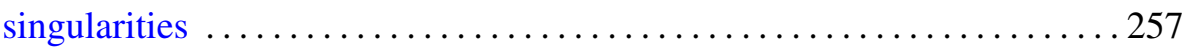

George E. Andrews, Multiple series Rogers-Ramanujan type identities . . . . 267

Didier Arnal, $*$ products and representations of nilpotent groups . . . . . . 285

David Cox and Walter Raymond Parry, Representations associated with

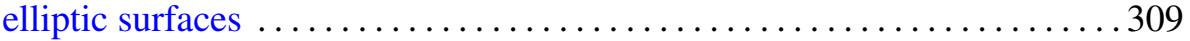

Joanne Marie Dombrowski, Tridiagonal matrix representations of cyclic

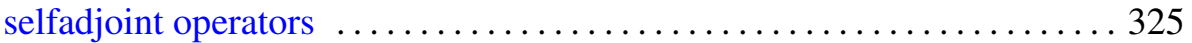

Ronald Dotzel, An Artin relation $(\bmod 2)$ for finite group actions on

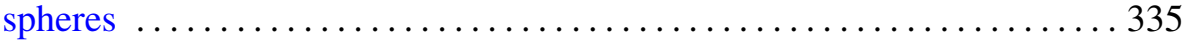

Leo Egghe, Convergence of adapted sequences of Pettis-integrable

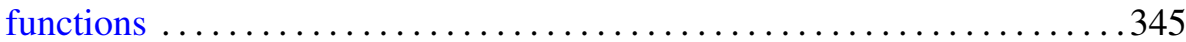

Rebecca A. Herb, Characters of induced representations and weighted

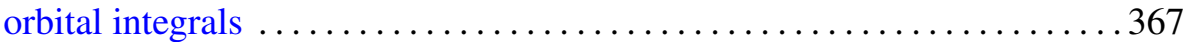

Steven M. Kahn, Cobordism obstructions to fibering manifolds over

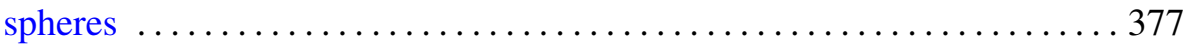

Robert D. Little, Projective space as a branched covering of the sphere with orientable branch set

Claude Schochet, Topological methods for $C^{*}$-algebras. III. Axiomatic

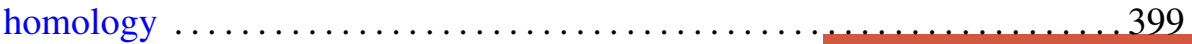

Claude Schochet, Topological methods for $C^{*}$-algebras. IV. $\bmod p$ homology

James M. Stormes, On the $K O$-orientability of complex projective varieties

Josephine Anne Ward, Characterization of homogeneous spaces and their norms 